\title{
Quantum Physics: A Doorway for the Potential Treatment of Cancer
}

\author{
Maria Shahzadi ${ }^{1}$, Muhammad Bilal ${ }^{2}$ \\ 1,2 Lecturer, Department of Health Professional Technologies, The University of Lahore
}

Dear Editor,

Questions exist regarding the scope of quantum physics and its potential applications. This field is so vast that someday, with little strife, it might come true that almost all the walks of life are entwined with quantum studies. Quantum physics has the potential to revolutionize medicine especially after the observation of some non-trivial phenomena of quantum mechanics like quantum tunnelling and superposition, being followed in living organisms at the cellular and molecular levels. ${ }^{1}$ Quantum physics has proven a foundational cornerstone in technological advancements. It's a novel field with the potential to morph our lives. ${ }^{2}$

Researchers are working on the development of quantum biological models to have a better understanding of biological activities, which include genetic defects, poor protein biosynthesis and inefficiencies in transmitted procedures like translation and transcription. Models in cancer research have benefited from concepts like quantum metabolism and entropy.

\section{Correspondence:}

Maria Shahazadi

Email: mariashahzadi58993@gmail.com

Cite this editorial: Shahzadi M, Bilal M. Quantum Physics:

A Doorway for the Potential Treatment of Cancer. J

Islamabad Med Dental Coll. 2021; 10(4):

Doi:
Over generations, cancer has developed by quantum-selective adjustments in the environment. Telomere length loss triggers a response to DNA mutation, resulting in senescence and the breakdown of cell checkpoints, which would otherwise kill cancer cells. ${ }^{3}$

Oncological applications of quantum physics are reflected as game-changers in a variety of domains. Quantum dots technology and quantum cascade laser technologies employ a variety of quantum physics principles that aid in the detection, imaging and treatment of cancerous cells which is otherwise challenging to do by using other fluorescent dyes such as green indocyanine, 5-aminolevulinic acid, and methylene blue. The principles of quantum physics have the potential to be deployed in the future to diagnose and treat cancer. ${ }^{4}$

The relevance of telomere shortening in cancer cells is uncertain, although it is conceivable that it triggers conditions that encourage or hinder cancer cell development. However, Quantum mechanics can be considered to demonstrate cancer cell proliferation.4 Medical community must pay attention to quantum physics which might play a strong role as a stakeholder to treat cancer at quantum levels. ${ }^{5}$

Keywords: Cancer treatment, Quantum physics, Quantum physics principles

\section{Acknowledgement:}

Authors would like to acknowledge our colleague Mr. Taimoor Hassan for guiding us in writing and manuscript submission. 


\section{References}

1. Faramarzpour $M$, Ghaderinia $M$, Abadijoo $H$, Aghababa $\mathrm{H}$. The Possibility of Quantum Medicine in Cancer Research: A Review. Biophysical Reviews and Letters. 2021:1-20. Doi: $10.1142 / \mathrm{S} 1793048021300012$

2. Raghunandan R, Voll M, Osei E, Darko J, Laflamme $R$. A review of applications of principles of quantum physics in oncology: do quantum physics principles have any role in oncology research and applications? Journal of Radiotherapy in Practice. 2019;18(4):383-94.Doi: $10.1017 /$ S1460396919000153
3. Uthamacumaran A. A biophysical approach to cancer dynamics: quantum chaos and energy turbulence. BioSystems.2017; 156-157: 1-22. Doi: 10. 1016/j.biosystems.2017.03.004.

4. Jacobson JI. A quantum theory of disease, including cancer and aging. Integr Mol Med. 2016 ;3(1):52441. Doi: 10.15761/IMM.1000200

5. Goh BH, Tong ES, Pusparajah P. Quantum Biology: Does quantum physics hold the key to revolutionizing medicine? Progress in Drug Discovery \& Biomedical Science. 2020;3(1). Doi:10.36877/pddbs.a0000130 